\title{
Recent results on Kaon Physics at KLOE-2
}

\author{
Alessandro Di Cicco ${ }^{1,2, *}$ on behalf of the KLOE-2 Collaboration \\ ${ }^{1}$ NFN Sezione di Roma Tre, Via della Vasca Navale 84, Rome, Italy \\ ${ }^{2}$ Dipartimento di Matematica e Fisica, Università Roma Tre, Via della Vasca Navale 84, Rome, Italy
}

\begin{abstract}
KLOE-2 extends the physics program of the forerunner KLOE experiment, especially in the field of discrete symmetries tests with neutral kaons. KLOE and KLOE-2 have collected together the largest sample of electronpositron collisions at an energy equal to the $\phi$-meson mass, corresponding to about $2.4 \times 10^{10}$ produced $\phi$ mesons.

The latest results on neutral kaon physics at KLOE will be reviewed, together with the status and prospects of the analyses of KLOE-2 data. A new measurement of the charge asymmetry in $\mathrm{K}_{\mathrm{S}}$ semileptonic decays with $1.7 \mathrm{fb}^{-1}$ of KLOE data, which improves the sensitivity of previous measurements of about a factor two, will be presented. Furthermore, the status of the analysis devoted to directly test $\mathcal{T}$ and $C \mathcal{P} \mathcal{T}$ symmetries in neutral kaons transitions, as well as the search of the pure $C \mathcal{P}$-violating $\mathrm{K}_{\mathrm{S}} \rightarrow 3 \pi^{0}$ decay using part of the recently acquired KLOE-2 dataset, will be presented.
\end{abstract}

\section{Introduction}

Flavoured neutral mesons are a powerful tool for testing fundamental discrete symmetries and their combinations such as $C \mathcal{P}$ and $C \mathcal{P T}$. Among them, neutral kaons are extensively used to search for manifestations of symmetry violations as they are easily translated to constraints on the properties of the mesons [1].

The KLOE experiment has contributed to determine most of the properties of neutral kaons as well as a number of tests of $C \mathcal{P}$ and $C \mathcal{P T}$ symmetries [2]. The KLOE dataset is still being analysed with the aim of obtaining more precise results on the charge asymmetry in semileptonic $\mathrm{K}_{\mathrm{S}}$ decays and preforming a first direct $C \mathcal{P T}$ test in transitions of neutral kaons. Furthermore, the KLOE-2 experiment has ended its data taking on March 2018, collecting about $5 \mathrm{fb}^{-1}$ of data, which will allow to enhance the upper limit set on the branching fraction

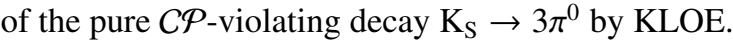

\section{KLOE and KLOE-2 experiments}

KLOE and KLOE-2 have been operated at the DA $\Phi N E \phi$-factory [3-5], which provided electron-positron collisions at the $\phi$ meson mass peak, $\sqrt{s} \approx 1020 \mathrm{MeV}$. $\phi$-meson decays into neutral kaons pairs - occurring with a branching fration of about $34 \%$ - were recorded by the KLOE apparatus, consisting of a huge cylindrical drift chamber (DC) [6], whose $\sim 50 \mathrm{~m}^{3}$

\footnotetext{
*e-mail: alessandro.dicicco@lnf.infn.it
} 
volume is filled with Helium (90\%) and Isobutane (10\%), surrounded by a lead/scintillatingfiber sampling calorimeter (EMC), whose coverage is about $98 \%$ of the solid angle [7].

KLOE-2 additionally featured a cylindrical triple-GEM inner tracker [8] - installed close to the $e^{+} e^{-}$interaction point to improve vertex reconstruction for quantum interferometry studies [9] - and a system of calorimeters, devoted to enlarge the solid angle coverage of the $\mathrm{EMC}$ for detecting photons from $\mathrm{K}_{\mathrm{S}, \mathrm{L}}$ decays and electrons/positrons from $\gamma-\gamma$ processes.

KLOE and KLOE-2 datasets provide the largest sample of $e^{+} e^{-} \rightarrow \phi$ events, corresponding to about 24 billions of produced $\phi$ mesons.

\section{$3 C P \mathcal{T}$ symmetry test with $\mathrm{K}_{\mathrm{S}}$ semileptonic decays}

A test of $C \mathcal{P T}$ symmetry can be performed by comparing the value of the lepton charge asymmetry, $A_{S, L}$, for short- and long-lived neutral kaons:

$$
A_{S, L}=\frac{\Gamma\left(K_{S, L} \rightarrow \pi^{-} e^{+} v\right)-\Gamma\left(K_{S, L} \rightarrow \pi^{+} e^{-} \bar{v}\right)}{\Gamma\left(K_{S, L} \rightarrow \pi^{-} e^{+} v\right)+\Gamma\left(K_{S, L} \rightarrow \pi^{+} e^{-} \bar{v}\right)}=2\left[\mathfrak{R}(\epsilon) \pm \mathfrak{R}(\delta)-\mathfrak{R}(y) \pm \mathfrak{R}\left(x_{-}\right)\right],
$$

where $\mathfrak{R}(\epsilon)$ and $\mathfrak{R}(\delta)$ are related to $\mathcal{T}$ and $C \mathcal{P T}$ violation in the $\mathrm{K}^{0}-\overline{\mathrm{K}^{0}}$ mixing, respectively, while $\mathfrak{R}(y)$ and $\mathfrak{R}\left(x_{-}\right)$parametrize the $C \mathcal{P T}$ violation in $\Delta S=\Delta Q$ and $\Delta S \neq \Delta Q$ decay amplitudes, respectively [10]. If $C \mathcal{P T}$ symmetry is not violated, then the aforementioned asymmetries are expected to be identical: $A_{S}=A_{L}=2 \mathfrak{R}(\epsilon) \simeq 3 \times 10^{-3}$.

The charge asymmetry for $\mathrm{K}_{\mathrm{L}}$ was precisely determined from the $\mathrm{KTeV}$ experiment at Fermilab: $A_{L}=\left(3.322 \pm 0.058_{\text {stat }} \pm 0.047_{\text {syst }}\right) \times 10^{-3}$ [11], while the most precise measurement of $A_{S}$ was performed by the KLOE collaboration using $410 \mathrm{pb}^{-1}$ of integrated luminosity collected in 2001-2002: $A_{S}=\left(1.5 \pm 9.6_{\text {stat }} \pm 2.9_{\text {syst }}\right) \times 10^{-3}[12]$.

The KLOE-2 Collaboration has recently published a new measurement based on a larger data sample, corresponding to an integrated luminosity of $1.63 \mathrm{fb}^{-1}$ collected in 2004$2005[13]: A_{S}=\left(-4.9 \pm 5.7_{\text {stat }} \pm 2.6_{\text {syst }}\right) \times 10^{-3}$, which improves the statistical accuracy of previous determination by almost a factor of two. Taking into account the correlation between both measurements, the combination of KLOE and KLOE-2 results is:

$$
A_{S}=\left(-3.8 \pm 5.0_{\text {stat }} \pm 2.6_{\text {syst }}\right) \times 10^{-3} \text {, }
$$

which represent the most precise measurement of $A_{S}$ to date. This value, combined with $\mathrm{KTeV}$ result on $A_{L}$ and providing also $\mathfrak{R}(\delta)$ [14] and $\mathfrak{R}(\epsilon)$ [15] as external inputs, allows to extract the $C \mathcal{P T}$-violating parameters [13]:

$$
\begin{aligned}
\mathfrak{R}\left(x_{-}\right) & =(-2.0 \pm 1.4) \times 10^{-3}, \\
\mathfrak{R}(y) & =(1.7 \pm 1.4) \times 10^{-3},
\end{aligned}
$$

which are consistent with $C \mathcal{P T}$ invariance and improve by almost a factor of two the previous results [12].

The rare $\mathrm{K}_{\mathrm{S}}$ decays are currently being studied using the full KLOE-2 dataset: with some $5 \mathrm{fb}^{-1}$ total integrated luminosity a pure data sample of about $5 \times 10^{9}$ tagged $\mathrm{K}_{\mathrm{S}}$ decays will be available.

\section{Direct test of $\mathcal{T}$ and $C \mathcal{P T}$ in transitions of neutral kaons}

The comparison of the rates of neutral mesons transitions between their flavour and $C \mathcal{P}$ eigenstates allows for a model independent test of $\mathcal{T}$ and $C \mathcal{P T}$ symmetries [16, 17]. Such a test - 
up to date performed only in the case of neutral B mesons, where it delivered the first direct evidence of $\mathcal{T}$ violation [18] - is pursued with the $\mathrm{K}^{0}-\overline{\mathrm{K}^{0}}$ system at KLOE-2. To this extent, quantum entangled meson pairs are used to identify the initial state of a particle transition by the decay of its entangled partner, while the final state is tagged by semileptonic and hadronic decays into two and three pions.

Two $\mathcal{T}$ violating observables are determined as ratios between the rates of two classes of processes: $\mathrm{K}_{\mathrm{S}} \mathrm{K}_{\mathrm{L}} \rightarrow \pi^{ \pm} e^{\mp} v, 3 \pi^{0}$ and $\mathrm{K}_{\mathrm{S}} \mathrm{K}_{\mathrm{L}} \rightarrow \pi^{+} \pi^{-}, \pi^{ \pm} e^{\mp} v$ :

$$
\begin{aligned}
& \mathrm{R}_{2}(\Delta t)=\frac{\mathrm{P}\left[\mathrm{K}^{0}(0) \rightarrow \mathrm{K}_{-}(\Delta t)\right]}{\mathrm{P}\left[\mathrm{K}_{-}(0) \rightarrow \mathrm{K}^{0}(\Delta t)\right]} \sim \frac{\mathrm{I}\left(\pi^{+} e^{-} \bar{v}, 3 \pi^{0} ; \Delta t\right)}{\mathrm{I}\left(\pi^{+} \pi^{-}, \pi^{-} e^{+} v ; \Delta t\right)}, \\
& \mathrm{R}_{4}(\Delta t)=\frac{\mathrm{P}\left[\overline{\mathrm{K}^{0}}(0) \rightarrow \mathrm{K}_{-}(\Delta t)\right]}{\mathrm{P}\left[\mathrm{K}_{-}(0) \rightarrow \overline{\mathrm{K}^{0}}(\Delta t)\right]} \sim \frac{\mathrm{I}\left(\pi^{-} e^{+} v, 3 \pi^{0} ; \Delta t\right)}{\mathrm{I}\left(\pi^{+} \pi^{-}, \pi^{+} e^{-} \bar{v} ; \Delta t\right)},
\end{aligned}
$$

where $\mathrm{I}\left(f_{1}, f_{2} ; \Delta t\right)$ denotes the number of recorded events characterized by a time-ordered pair of kaon decays $f_{1}$ and $f_{2}$ separate by an interval of proper kaon decay times $\Delta t$ [16]. Any deviations of the asymptotic level of these ratios from unity for large transition times would be a manifestation of $\mathcal{T}$ violation.

The novel concept of such test can be generalized to test $C \mathcal{P T}$ symmetry through the determination of the asymptotic level of the following double ratio:

$$
\frac{R_{2}^{C P T}}{R_{4}^{C P T}}=\frac{\mathrm{P}\left[\mathrm{K}^{0}(0) \rightarrow \mathrm{K}_{-}(\Delta t)\right] / \mathrm{P}\left[\mathrm{K}_{-}(0) \rightarrow \overline{\mathrm{K}^{0}}(\Delta t)\right]}{\mathrm{P}\left[\overline{\mathrm{K}^{0}} \rightarrow \mathrm{K}_{-}(\Delta t)\right] / \mathrm{P}\left[\mathrm{K}_{-}(0) \rightarrow \mathrm{K}^{0}(\Delta t)\right]} \stackrel{\Delta t \gg \tau_{S}}{=} 1-8 \mathfrak{R} \delta-8 \mathfrak{R} x_{-},
$$

where the $\delta$ and $x_{-}$are the parameters already presented in Equation 1. This double ratio constitutes a robust $C \mathcal{P T}$-violation-sensitive observable [17], which has never been measured.

The analysis tools required to determine both the $\mathcal{T}$-violation-sensitive ratios and the $C \mathcal{P T}$-violation-sensitive double ratio are being tested using the full KLOE dataset, corresponding to an integrated luminosity of about $1.7 \mathrm{fb}^{-1}$. A preliminary distribution of the ratio defined in Equation 6 is presented in Figure 1. Although the uncertainty on the asymptotic level of these observables available with KLOE data is only at the percent level, the analysis of the full KLOE-2 dataset will allow to perform statistically significant tests of $\mathcal{T}$ and $C \mathcal{P T}$ at the $10^{-3}$ level of precision.

\section{Search for the pure $C \mathcal{P}$-violating $\mathrm{K}_{\mathrm{S}} \rightarrow 3 \pi^{0}$ decay}

Another class of $C \mathcal{P}$-violating phenomena in the neutral kaon system is represented by the

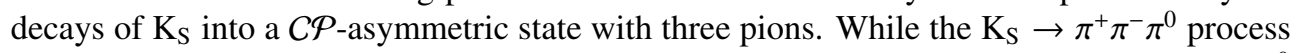
contains both $C \mathcal{P}$-violating and conserving amplitudes, the observation of the $\mathrm{K}_{\mathrm{S}} \rightarrow 3 \pi^{0}$

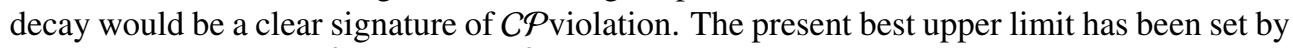
$\mathrm{KLOE}$ at $\mathrm{BR}\left(\mathrm{K}_{\mathrm{S}} \rightarrow 3 \pi^{0}\right)<2.6 \times 10^{-8}$ [19], still almost an order of magnitude lower than the precision of the Standard Model prediction $\left(1.9 \times 10^{-9}\right)$. Therefore, the search for $\mathrm{K}_{\mathrm{S}} \rightarrow 3 \pi^{0}$ is already performed using the recently collected $\sim 5 \mathrm{fb}^{-1}$ of KLOE-2 data. With a larger data sample and an optimized analysis to properly treat KLOE-2 data, the sensitivity on this decay measurement is expected to be beyond the level of $10^{-8}$.

\section{Summary}

The measurements of the KLOE detector have recently delivered the most precise result on the charge asymmetry in the semileptonic decays of the short-lived neutral $\mathrm{K}$ meson, 


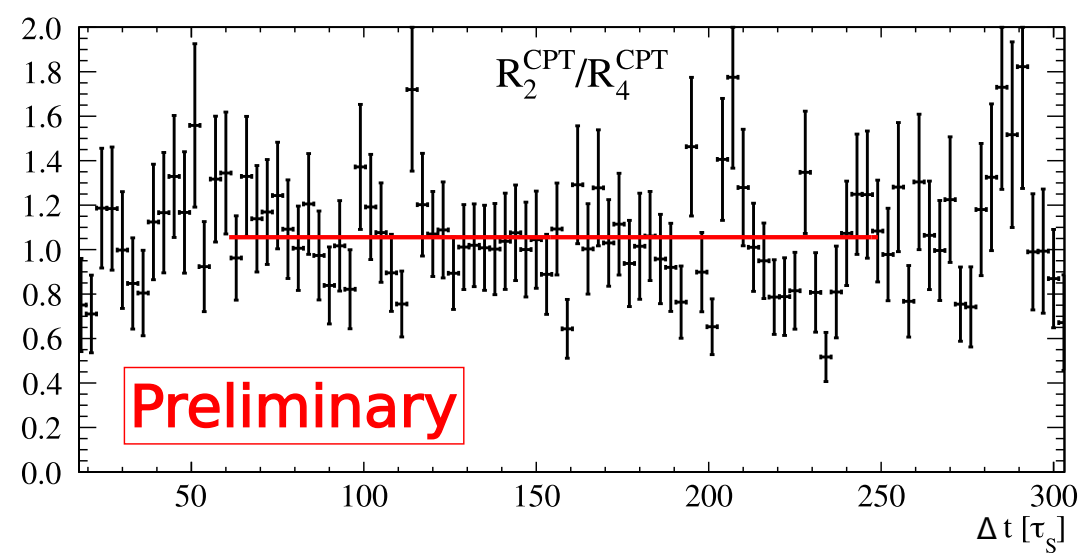

Figure 1. Preliminary distribution of the $C \mathcal{P T}$ violation-sensitive double ratio of neutral kaon double decay rates as a function of the time difference between entangled kaon decay times $(\Delta t)$. The statistical uncertainty of the asymptotic level of this observable for $\Delta t \gg \tau_{S}$ (red line) amounts to 0.011 using the full KLOE dataset.

consistent with $C \mathcal{P T}$ conservation within the total uncertainty of $5.6 \times 10^{-3}$. The larger dataset collected by the upgraded KLOE-2 detector is expected to further improve the precision of this measurement to the level of $3 \times 10^{-3}$. The full datasets of KLOE is being used to perform a first direct tests of $\mathcal{T}$ and $C \mathcal{P T}$ symmetries in transitions of neutral kaons. Profiting also of KLOE-2 data, the precision on this novel tests is expected to reach the level of $10^{-3}$. Finally, the search for the pure $C \mathcal{P}$-violating decay $\mathrm{K}_{\mathrm{S}} \rightarrow 3 \pi^{0}$ is being performed with the full KLOE2 dataset: The expected sensitivity to this process should allow KLOE-2 Collaboration to approach the level of the present Standard Model predicion for $\mathrm{BR}\left(\mathrm{K}_{\mathrm{S}} \rightarrow 3 \pi^{0}\right)$.

We warmly thank our former KLOE colleagues for the access to the data collected during the KLOE data taking campaign. We thank the DAФNE team for their efforts in maintaining low background running conditions and their collaboration during all data taking. We want to thank our technical staff: G.F. Fortugno and F. Sborzacchi for their dedication in ensuring efficient operation of the KLOE computing facilities; M. Anelli for his continuous attention to the gas system and detector safety; A. Balla, M. Gatta, G. Corradi and G. Papalino for electronics maintenance; C. Piscitelli for his help during major maintenance periods. This work was supported in part by the Polish National Science Centre through the Grants No. 2013/11/B/ST2/04245, 2014/14/E/ST2/00262, 2014/12/S/ST2/00459, 2016/21/N/ST2/01727, 2016/23/N/ST2/01293, 2017/26/M/ST2/00697.

\section{References}

[1] A. Di Domenico, in Handbook on neutral kaon interferometry at a $\Phi$-factory (INFN LNF - Divisione Ricerca - SIS - Ufficio Pubblicazioni, P.O. Box 13, I-00044 Frascati, Rome, Italy, 2007)

[2] F. Bossi, E. De Lucia, J. Lee-Franzini, S. Miscetti, M. Palutan, Riv. Nuovo Cim. 31531 (2008)

[3] A. Gallo et al., Conf. Proc. C 060626604 SLAC-PUB-12093 (2006)

[4] M. Zobov et al., Phys Rev. Lett. 104174801 (2010)

[5] C. Milardi et al., JINST 7 T03002 (2012) 
[6] M. Adinolfi et al., Nucl. Inst. Meth. A 48851 (2002)

[7] M. Adinolfi et al., Nucl. Inst. Meth. A 482364 (2002)

[8] G. Bencivenni et al., JINST 12 C07616 (2017)

[9] G. Amelino Camelia et al., Eur. Phys. J. C 68619 (2010)

[10] L. Maiani, in The Second DAФNE Physics Handbook Vol. I p. 3 (INFN - LNF Frascati, Rome, Italy, 1995)

[11] A. Alavi-Harati et al., Phys. Rev. Lett. 88181601 (2002)

[12] F. Ambrosino et al., Phys. Lett. B 636173 (2006)

[13] A. Anastasi et al., Accepted at: JHEP 0921 (2018)

[14] C. Patrignani, et al. (Particle Data Group), Chin. Phys. C 40100001 (2016)

[15] F. Ambrosino et al., JHEP 1211 (2006)

[16] J. Bernabeu, A. Di Domenico, P. Villanueva-Perez, Nucl. Phys. B 868102 (2013)

[17] J. Bernabeu, A. Di Domenico, P. Villanueva-Perez, JHEP 10139 (2015)

[18] J. P. Lees et al., Phys. Rev. Lett. 109211801 (2012)

[19] D. Babusci et al., Phys. Lett. B 72354 (2013) 